\title{
Electromyographic Signal Compression Through Image Encoders and Preprocessing Techniques
}

\author{
Wheidima C. de Melo, Eddie B. de Lima Filho, and Waldir S. da Silva Júnior
}

\begin{abstract}
Recently, two-dimensional techniques were successfully employed for encoding surface electromyographic (S-EMG) records, through the use of off-the-shelf image encoders as an effective alternative for that kind of signal. However, as SEMG signals are very different from natural images, there is often a preprocessing step before compression, in an attempt to improve the performance of the chosen encoder. This paper address the mentioned approach and presents an investigation regarding the performance of video and image encoders, when used for compressing S-EMG signals. In addition, two new preprocessing techniques are introduced, named as euclidean distance sorting (EDS) and region-based euclidean distance sorting (REDS), which have the potential to enhance the exploitation of intersegment correlations, normally present on S-EMG records. Experiments were carried out with real isometric records acquired in laboratory, which were firstly preprocessed and then compressed with the JPEG2000, H.264/advanced video coding, and high efficiency video coding (HEVC) algorithms. A brief analysis reveals that the proposed scheme is effective, given that JPEG2000 and HEVC allied to EDS and REDS even outperform state-of-the-art schemes available in the literature, in terms of PRD $\times$ Compression Ratio and spectral-parameter estimation.
\end{abstract}

Index Terms-S-EMG, HEVC, surface electromyography, image compression.

\section{INTRODUCTION}

C URRENTLY, surface electromyographic (S-EMG) signals are an interesting approach for studying and diagnosing muscle-related pathologies [2], [3], [4], such as nerve compression or injury, amyotrophic lateral sclerosis, and muscular dystrophy, monitoring patients, activating control interfaces [5], and even predicting pre-term labor [6], due to its non-invasive behavior. Indeed, they have been attracting attention in the past few years, mainly due to the development of new digital signal processing techniques, which provide new paradigms and applications. For instance, S-EMG signals can be used for classifying finger movements, through linear discriminant analysis [7]. Besides, the generation of realistic S-EMG signals is also an interesting area, whose goal is to

The Associate Editors coordinating the review of this manuscript and approving it for publication were Profs. Ceclio Pimentel and Marcelo da Silva Pinho.

W. C. de Melo is with State University of Amazonas, Av. Darcy Vargas, 1200, Parque 10,69050-020, Manaus-AM, Brazil, e-mail: wmelo@uea.edu.br.

W. S. da Silva Júnior is with Federal University of Amazonas, Av. General Rodrigo Octávio, 6200, Coroado I, 69077-000, Manaus-AM, Brazil, e-mail: waldirjr@ufam.edu.br.

E. B. de Lima Filho is with Federal University of Amazonas, Av. General Rodrigo Octávio, 6200, Coroado I, 69077-000, Manaus-AM, Brazil, and FPF Tech, Av. Governador Danilo de Matos Areosa, 1170, Distrito Industrial, 69075-351, Manaus-AM, Brazil, e-mail: eddie.filho@fpf.br.

A preliminary version of this paper was presented in XXXIII Simpsio Brasileiro de Telecomunicaes (SBrT'15), Juiz de Fora, MG, Brazil, September 1-4, 2015 [1].

Digital Object Identifier: 10.14209/jcis.2016.17 provide a mean for evaluating parameter-estimation algorithms and a better understanding regarding the relationship between such parameters and biological or physiological characteristics [8].

S-EMG signals record the electrical activity responsible for controlling muscle contractions in human bodies, that is, they directly represent electrical impulses sent by human nervous systems to muscle fibers. It is worth noticing that S-EMG signals are present even when a limb is lost [9], [10], which can be used for triggering similar actions.

When acquiring S-EMG signals, which generally needs specific hardware and circuitry [11] that must also produce a signal with acceptable quality [12], one has to chose parameters such as sampling rate, bit depth, number of channels (derivations), and record duration, which have direct impact on necessary transmission and storage resources. In addition, such a problem is even worse when high-density electromyography [13] is employed, due to the large amount of data. In summary, S-EMG signal compression is a bandwidth-demading technique that consequently raises the need for efficient compression methods, which besides producing a more compact data representation, must also be able to preserve the associated clinical/useful information [2], [3], [6], [14], [15].

Although S-EMG records are originally one-dimensional, some algorithms have employed a different approach for their compression: processing the related data as two-dimensional signals [16], [17], by splitting them into segments that occupy columns or rows of an image. This way, the input signal is preprocessed and then passed to an image/video compressor, which is in charge of exploiting intra and intersegment redundancies.

This article addresses the mentioned problem and proposes a methodology for compressing S-EMG signals as images, with results for three different two-dimensional back ends: JPEG2000 [18], [19], H.264/advanced video coding (AVC) [20], and high efficiency video coding (HEVC) [21]. Besides, two new preprocessing techniques are introduced, named as euclidean distance sorting (EDS) and region-based euclidean distance sorting (REDS), whose goal is to improve the exploitation of intersegment dependencies, that is, redundancy among image columns or rows.

Finally, the current text is a fully revised and extended version of the work presented by Melo, Filho, and Júnior [1], which now includes a new preprocessing technique (REDS) and associated simulations, a related work section, a brief discussion about HEVC, and results regarding preservation of spectral parameters and computational complexity (encoding times).

The remainder of this paper is organized as follows. In 
Section II, the related work is presented. Section III, in turn, introduces the new preprocessing techniques: EDS and REDS. In Section IV, the proposed compression scheme is presented, in such a way that the role of each component is briefly explained. Experimental results are provided in Section V, with actual S-EMG signals recorded in laboratory, and, finally, Section VI draws the conclusions of the present work.

\section{RELATED WORK}

Traditionally, S-EMG signals are handled and compressed as one-dimensional sources. Besides, regarding inner codingmachines, as happens to other biological signals [14], S-EMG signal compressors can be classified into three distinct groups: direct, parametric, and transform-based.

Direct approaches generally estimate signal samples, whose resulting residue is then quantized and possibly encoded. Examples of such a group are the scheme developed by Norris and Lovely [22], which is based on adaptive differential pulse code modulation with adaptive quantization, and the one introduced by Grönfors, Reinikainen, and Sihvonen [23], which employs mean residual vector quantization with short segments.

Parametric schemes, in turn, extract features from input records, which are later used for signal reconstruction, as the one reported by Carotti et al. [24]. In summary, their technique is based on autoregressive models and is able to preserve SEMG spectral features.

In the transform-based group, input signals are transformed to another domain, where they are quantized and entropy coded. Indeed, if the combination between transformation and coding is carefully chosen, high performance can be achieved [14]. There are many approaches and most use the discrete cosine transform (DCT) [15], [25] or wavelets [26], however, the latter generally present better results. For instance, Norris, Englehart, and Lovely [26] applied the embedded zero-tree wavelet algorithm to S-EMG segments of 1024 samples, Berger et al. [27] employed adaptive bit-allocation through an artificial neural network, Jain and Vig [28] used tree vectors for rearranging wavelet coefficients, which are then quantized, and, finally, Trabuco et al. [29], [30] proposed an algorithm that uses wavelet transform followed by dynamic bit allocation, based on mathematical decreasing spectral shape models for reducing the bit depth of high-frequency coefficients, and entropy coding.

Other approaches can be classified into two of the mentioned groups, as the one based on the multidimensional multiscale parser (MMP) algorithm [31], which tries to approximate S-EMG segments, of different lengths, by elements retrieved from an adaptive multiscale dictionary, through scale transforms.

Recently, a new trend has been noticed, regarding S-EMG compressors: algorithms that compress S-EMG signals as if they were two-dimensional, that is, S-EMG records compressed as images, with one [16], [17] or multiple electrodes [13], as show in Fig. 1. Indeed, those schemes may also be classified into one of the three mentioned groups, however, the majority of the algorithms developed so far are transformbased. Costa et al. [16] reorganizes S-EMG segments into matrix columns, which are then preprocessed and compressed by off-the-shelf image encoders: JPEG2000 [18], [19] and H.264/AVC [20]. Melo, Filho, and Júnior [17] proposed a similar scheme, besides two new preprocessing algorithms: the relative complexity sorting and the percentage difference sorting. In summary, such schemes showed that it is worth while pursuing new preprocessing techniques for enhancing the performance of commercial image encoders, given that the deployment of such an approach may be favored, since no new compressor is developed from scratch and the chosen encoder may already be available, in the target platform (e.g., personal computers, mobile platforms, etc.).

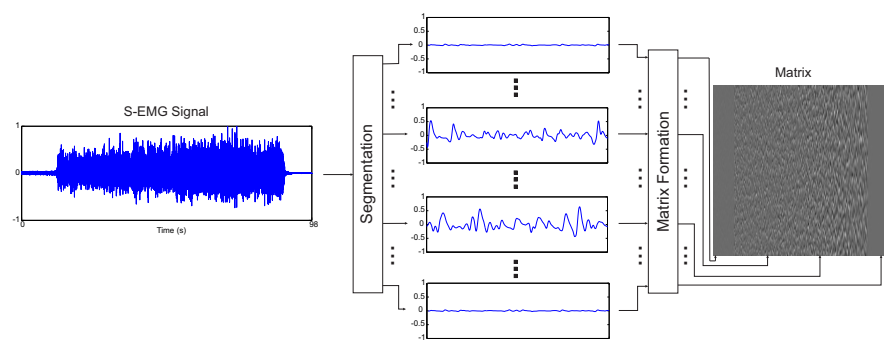

Fig. 1. An S-EMG signal reorganized as image.

The present approach follows the two-dimensional trend, introduces two new preprocessing techniques, and employs a new compressor: HEVC. Indeed, the latter is an interesting choice for S-EMG signal compression, given that it provides state-of-the-art results, regarding images, and presents flexible segmentation and prediction procedures, which may be useful for S-EMG signals, since they do not behave like natural images and their intra and intersegment redundancies may not be easily exploited. Indeed, natural (scene) images [32] are representations of scenes in everyday life, such as buildings, signs, and urban environments. They are characterized by highly-correlated neighbouring pixels, in all directions, which does not usually happen in images created with S-EMG signals.

\section{New Preprocessing Techniques}

Preprocessing techniques allow an effective use of twodimensional encoders, since the latter are often developed for natural images and, consequently, they may not be able to handle particularities of S-EMG signals. In summary, they reorganize signal segments, in such a way that two-dimensional tools are more effectively employed.

In the following subsections, the proposed preprocessing techniques will be presented, in detail.

\section{A. The Euclidean Distance Sorting Algorithm}

Image and video compressors are fine-tuned for natural images; however, such images are very different from S-EMG ones. Regarding the latter, input S-EMG records are segmented and reassembled into two-dimensional arrays, which initially look like noise, as can be seen in Fig. 2(a). Indeed, S-EMG signals can be modeled as Gaussian or Laplacian processes, depending on the force level [33]. Therefore, the primary goal 
of any preprocessing technique applied to S-EMG images is to generate a more encoder-friendly representation, so that twodimensional encoders are able to efficiently exploit the existing signal correlations.

In addition, it is worth noticing that the mentioned intermediate representation often incurs additional data. For instance, in order to use a preprocessing technique that reorders segments of the input signal, a given compression scheme must transmit side information, which contains the original position of each segment, so that the inverse procedure can be carried out, in the decoder end. Therefore, if a given technique does not efficiently increase signal dependencies, that is, in such a way that the additional data is compensated by the coding gain, the overall resulting performance may be lower than the original one.

The proposed preprocessing technique consists in an efficient reordering procedure called EDS, whose purpose is to reorganize signal segments based on their similarities. It is carried out according to the euclidean distance

$$
E D(x, y)=\sqrt{\sum_{n=0}^{N-1}(x[n]-y[n])^{2}},
$$

where $\operatorname{ED}(x, y)$ is the euclidean distance metric, computed for segments $x$ and $y, x[n]$ is the last sorted segment, $y[n]$ is the segment under analysis, and $N$ is the number of samples in each segment. The EDS procedure begins by placing the segment with smallest variance in the first column of the image matrix; then, the other segments are inserted into the remaining columns, according to their euclidean distances regarding the last sorted segment, which is computed with (1).

An example of the proposed technique is shown in Fig. 2(b). One can notice that the new S-EMG image presents a more organized texture, whose complexity increases from left to right, and larger homogeneous areas can be found on its left portion. As a consequence, two-dimensional encoders will be favored, which will also result in a more compact representation.

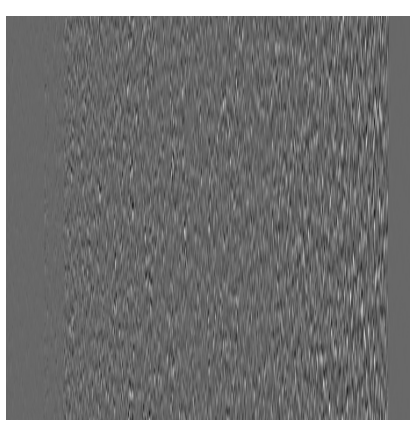

(a)

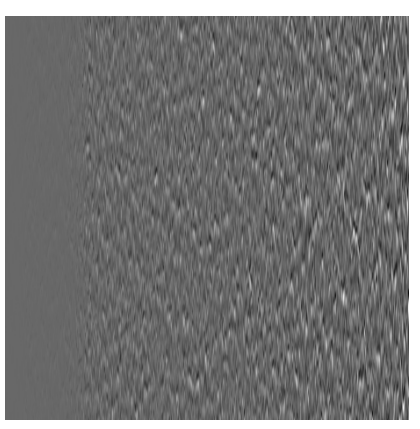

(b)
Fig. 2. Example regarding the EDS technique: (a) original matrix and (b) reordered matrix.

\section{B. The Region-Based Euclidean Distance Sorting Algorithm}

The EDS technique guarantees that similar segments become adjacent, which creates homogeneous regions and con- sequently favor decorrelation and prediction techniques performed by image encoders. In addition, some algorithms also divide input images into rectangular blocks, which are later predicted and even further segmented. For instance, H.264 employs $16 \times 16$ and $4 \times 4$ intra prediction blocks [20], while HEVC uses block sizes ranging from $4 \times 4$ to $32 \times 32$ [34]. However, during preprocessing, signal segments are reordered without taking into account input-block sizes and, consequently, even with EDS, signal blocks may present undesired discontinuities, which is more noticeable when compressing signals with high variance.

The discussion above was the inspiration for another preprocessing technique, named as REDS, which is shown in Fig. 3. The REDS procedure begins by finding the segment pair with smallest euclidean distance, according to (1), and placing the associated segments in the first two columns of the reordered matrix; the segment with smallest variance is the first one. The other segments are then inserted into the remaining columns, according to their euclidean distances regarding the last sorted segment, until 32 segments are relocated, which consists in a segment region. Next, the new segment pair with smallest euclidean distance, among the remaining elements, is found and the previous procedure is repeated.

Initially, the region size of 32 segments was chosen, in an attempt to provide a match for both H.264 and HEVC block sizes, that is, in order to ensure homogeneous regions across $32 \times 32$ block boundaries, as explained above. Nonetheless, many simulations were also performed, with regions of 64 , 32,16 , and 8 segments, using H.264 and HEVC, and the associated results showed a slight advantage for a group size of 32 segments. In addition, smaller regions led to worse results.

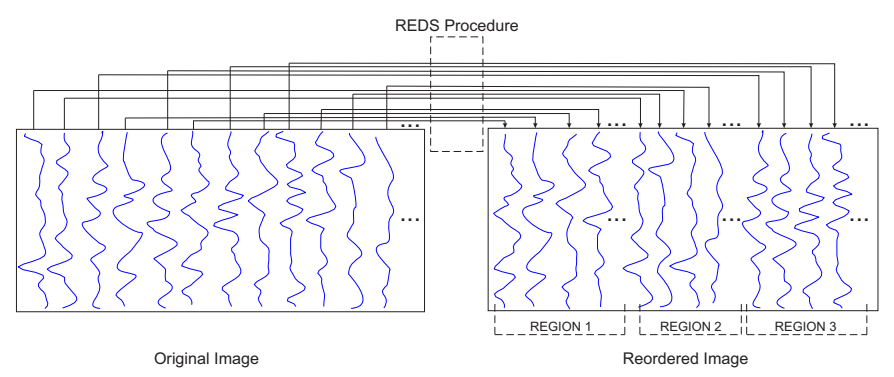

Fig. 3. Illustration of the REDS technique.

\section{The Proposed Compression Architecture}

The proposed compression scheme is composed of three steps, as shown in Fig. 4: matrix formation, preprocessing, and image compression. The matrix formation step splits the input S-EMG signal, so that the number of samples, in each segment, is equal to 128. This way, each segment is placed into a column of the new S-EMG matrix, from left to right. Next, the resulting matrix is reorganized, according to EDS or REDS, which generates a list of column indices that are arithmetically encoded [35] and then transmitted (e.g., in the file header), as side information. In the last step, the resulting two-dimensional signal is encoded with one of three available encoders: JPEG2000, H.264/AVC, or HEVC. At the 
decoder end, all presented steps are performed in reverse order (i.e., decoding, reordering into original form, and segment concatenation), as shown in Fig. 4, through the use of the transmitted side information, which bears the original segment positions.

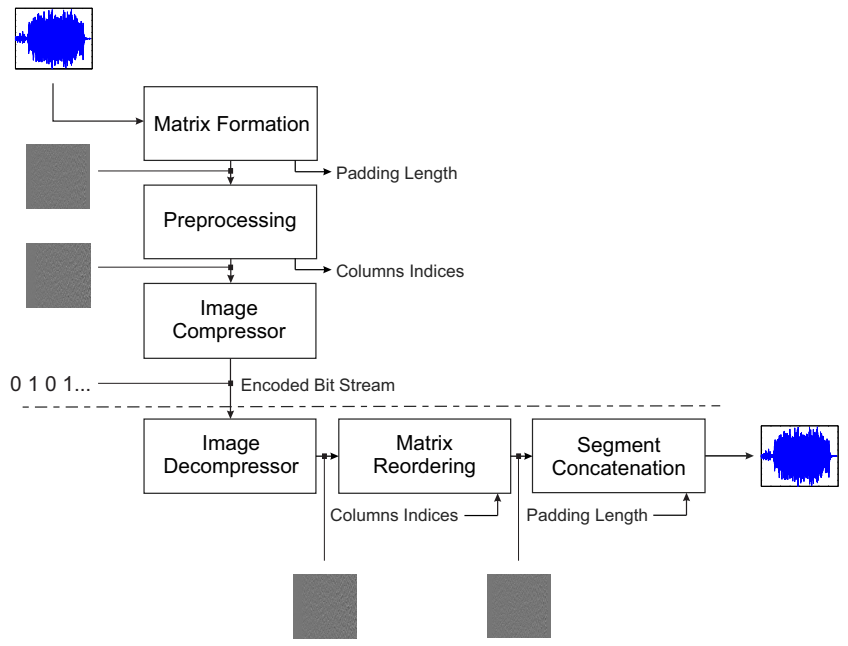

Fig. 4. Block diagram of the proposed architecture.

\section{A. HEVC as Back End for S-EMG Compression}

In this work, the HEVC encoder is introduced for compressing electromyographic signals. HEVC is a new standard for video coding, which provides great improvement when compared with its predecessor, that is, H.264/AVC [36]. Although HEVC has been designed for video compression applications, it can also be applied to still images, through the use of its intra coding tools. As a result, state-of-the-art performance is obtained, which is even better than what is presented by transform-based commercial image encoders [37].

As already mentioned, compression methods applied to S-EMG signals are traditionally based on one-dimensional techniques [27], [31], [38], [39]. However, some studies have proposed schemes based on JPEG2000 and H.264/AVC [16], [40], with competitive results. Therefore, an investigation of the HEVC performance, when compressing electromyographic signals, is justifiable and even necessary.

The HEVC image compression scheme consists of blockbased spatial prediction combined with transform-based coding [21]. HEVC initially splits input images into tiles (parallel encoding/decoding tool) and/or slices, which are further segmented into coding tree units (CTUs). CTUs are the basic coding units, such as macroblocks in H.264/AVC, and can be $16 \times 16,32 \times 32$, or $64 \times 64$. Each CTU can be recursively partitioned into smaller square regions named as coding units (CUs), using a quadtree structure, and CUs can be divided into prediction units (PUs) and a tree of transform units (TUs). PUs are used in prediction processes and TUs in transform and quantization procedures.

In order to exploit spatial redundancy, HEVC employs block-based prediction through PUs, which is performed using previously encoded samples from neighboring blocks. Such scheme presents 33 angular intra prediction modes, which are combined with block sizes ranging from $4 \times 4$ to $64 \times 64$. Furthermore, there are two non-angular prediction modes, named as DC and planar, which are used to exploit smooth image regions. The resulting residue signal is then converted to frequency domain with DCT; actually, the transform used in HEVC is a finite precision approximation of DCT [41]. In addition, HEVC also provides a $4 \times 4$ discrete sine transform. The output frequency coefficients are then quantized and entropy-coded, with the goal of exploiting their statistical correlation [21].

\section{EXPERIMENTAL RESULTS}

In order to evaluate the proposed scheme, tests with SEMG signals, acquired from the biceps brachii of 13 subjects, were performed. The mentioned signals were collected during isometric activities, where each subject remained seated, with his upper arm parallel to his torso and sustaining $60 \%$ of maximum voluntary contraction (MVC). The resulting records present durations ranging from 1.3 to 3.0 minutes and were digitized at $2000 \mathrm{~Hz}$, with a bit depth of 12 , which is common practice in this research area [16], [27], [31].

Each test signal was converted into an image and encoded by JPEG2000 (Kakadu version ${ }^{1}$ ), H.264/AVC (JM 18.6 reference software ${ }^{2}$ ), and HEVC (HM 16.2 reference software ${ }^{3}$ ). JPEG2000 was used with 0.000025 quantization step, bit depth of 16, and Cohen-Daubechies-Feauveau 9/7 wavelet kernel. H.264/AVC was configured in intraframe mode, with FRExt High 100 profile, rate-distortion optimization, context adaptive binary arithmetic coding, and deblocking filter. HEVC was employed in intraframe mode, with rate-distortion optimized quantization and deblocking filter.

\section{A. Results Regarding Reconstructed-Signal Quality}

The quality of reconstructed signals was evaluated with percent root-mean-square difference $(\mathrm{PRD}) \times$ compression factor $(\mathrm{CF})$ curves. The PRD metric is defined as

$$
P R D=\sqrt{\frac{\sum_{i=0}^{N-1}(x[i]-\hat{x}[i])^{2}}{\sum_{i=0}^{N-1} x^{2}[i]}} \times 100,
$$

where $x[i]$ is the original signal, $\hat{x}[i]$ is the reconstructed signal, and $N$ is the number of samples, in each signal. The CF metric is defined as

$$
C F=\frac{B_{o}-B_{c}}{B_{o}} \times 100,
$$

where $B_{o}$ is the total number of bits, in the original signal, and $B_{c}$ is the total number of bits, in the compressed version. As the bit depth (number of bits per sample) is equal to 12 , $B_{o}=12 \times N$.

\footnotetext{
${ }^{1}$ Available at http://www.kakadusoftware.com/downloads

${ }^{2}$ Available at http://iphome.hhi.de/suehring/tml/download/

${ }^{3}$ Available at https://hevc.hhi.fraunhofer.de/
} 
PRD $\times$ CF curves for the 13 test $\mathrm{S}-\mathrm{EMG}$ signals, using the proposed compression scheme with HEVC, JPEG2000, and H.264 allied to EDS and REDS, are presented in Fig. 5, along with an average reference for the entire set, while numerical average results are shown in Table I. One may observe that although there is no easily noticeable difference between the two proposed preprocessing techniques, a few specific records are favored by REDS, depending on the chosen encoder, such as in the fourth curve, from top to bottom (highlighted with a continuous line), in Fig.5(b) (see the same curve in Fig.5(a)). Indeed, the combination between REDS and HEVC provided slightly better results only with signals presenting high variance, such as the mentioned one. As a consequence, REDS may be an interesting option for more complex signals, when using HEVC; however, it also presents a slight loss of performance for less complex records, at high CFs (see Fig. 5 and Table I). Besides, since most records in the test set present small variance, the net result is an increasing advantage towards EDS, regarding average curves. Finally, the chosen test signals present curves with different slopes and results spreading over a dynamic range of $9 \%$ of $\mathrm{PRD}$, which corroborates their suitability for this kind of experiment.

Regarding HEVC and for compression factors below $86 \%$, the proposed scheme maintains acceptable PRD values $(<7 \%)$ [31], which is generally enough for not compromising the diagnostic procedure, as shown in Figs. 5(a) and 5(b). Besides, the resulting curves do not present large spread at high CFs, when compared with the ones provided by the other back ends.

The results for H.264/AVC allied to EDS and REDS provided, for CFs below $81 \%$, PRD figures below $7 \%$. One may also notice that the results in Fig. 5 and Table I are worse than what was presented for HEVC, which may appear strange, since they use similar approaches. The main reason behind such a behavior is that the compression procedure of HEVC is more flexible and includes many more prediction directions, when compared with H.264/AVC, which has the potential to further exploit the correlations available in S-EMG records.

Finally, the combination between JPEG2000 and EDS or REDS achieved PRD figures lower than $7 \%$, for CFs below $84 \%$. Clearly, there is a slight advantage at low CFs, when compared with HEVC, as shown in Table I, however, when CF increases, HEVC provides better results. Indeed, JPEG2000 is not very competitive at high CFs (low bit rates) [42] and its wavelet transform is favored by the large homogeneous areas created by the sorting procedure, which has a direct impact on the resulting coding efficiency.

On one hand, for high CFs, HEVC achieved very good results, where the associated difference increases for larger $\mathrm{CF}$ values, in comparison with the other tested encoders. On the other hand, JPEG2000 obtained the best performance for low CFs. The proposed scheme with H.264/AVC achieved the worst results, for the entire $\mathrm{CF}$ range.

Table I also provides average results for the base algorithms alone, without the EDS or REDS techniques. As one may notice, both techniques improved the exploitation of intersegment dependencies, which consequently increased the performance of each tested encoder. However, the performance gain for HEVC was only marginal, which means that, regarding iso- metric signals, only the matrix formation step may be enough, as can be concluded from the average results, although there are significant gains for specific records. In addition, there is a performance decrease for the combination between REDS and HEVC, for a CF of $90 \%$, which means that the coding gain due to reordering did not compensated the transmitted side information. For CFs below $85 \%$, the combination between REDS and JPEG2000 achieved the best results, while HEVC combined with REDS and EDS overcame all combinations for CFs of $85 \%$ and $90 \%$, respectively. It is worth noticing that the combination between EDS/REDS and JPEG2000 overcame HEVC, for a $\mathrm{CF}$ of $80 \%$, which happened only due to the developed preprocessing techniques.

TABLE I

AVERAGE $P R D(\%)$ RESUlTS FOR THE PROPOSED SCHEME, WITH AND

\begin{tabular}{cccccc}
\multicolumn{6}{c}{ WITHOUT THE EDS AND REDS TECHNIQUES. } \\
\hline \hline Compression Factor & $70 \%$ & $75 \%$ & $80 \%$ & $85 \%$ & $90 \%$ \\
\hline JPEG2000 & 1.016 & 1.552 & 2.402 & 4.004 & 7.913 \\
EDS + JPEG2000 & 0.955 & 1.409 & 2.201 & 3.698 & 7.445 \\
REDS+JPEG2000 & $\mathbf{0 . 9 5 3}$ & $\mathbf{1 . 4 0 8}$ & $\mathbf{2 . 2 0 0}$ & 3.745 & 7.500 \\
H.264/AVC & 2.182 & 2.879 & 3.943 & 5.861 & 9.884 \\
EDS + H.264/AVC & 1.909 & 2.491 & 3.466 & 5.342 & 9.273 \\
$R E D S+$ H.264/AVC & 1.903 & 2.500 & 3.479 & 5.361 & 9.356 \\
HEVC & 1.365 & 1.639 & 2.254 & 3.438 & 6.266 \\
EDS+HEVC & 1.343 & 1.634 & 2.234 & 3.417 & $\mathbf{6 . 2 3 8}$ \\
$R E D S+$ HEVC & 1.347 & 1.601 & 2.220 & $\mathbf{3 . 4 0 8}$ & 6.271 \\
\hline
\end{tabular}

Average results for state-of-the-art schemes, available in the literature, as well as results obtained with HEVC, JPEG2000, and H.264/AVC allied to EDS and REDS are shown in Table II. The proposed approach outperformed the other evaluated methods, for CFs of $75 \%$ and $80 \%$, using REDS and JPEG2000, and also for a CF of 90\%, using EDS and HEVC, besides being competitive regarding the state-of-the-art, for a CF of $85 \%$, with REDS and HEVC.

As already mentioned, the methods presented by Norris, Englehart, and Lovely [38], Berger et al. [27], and Trabuco et al. [29], [30] are based on wavelets, while the one introduced by Filho, Silva, and Carvalho [31] employs a spatial domain approach, which approximates signal segments with elements from an adaptive dictionary. The scheme presented by Trabuco et al. overcame the proposed method only for a CF of $85 \%$, which probably happened due to the high performance of its bit allocation scheme, for this specific CF. It is worth noticing that although the approach presented by Costa et al. [16] is similar to the one proposed here, even the results provided by the same compressors, without preprocessing, are superior, which indicates that the matrix formation used here is superior or the encoder configuration was not thoroughly performed.

In summary, HEVC allied to preprocessing techniques presented good results for CFs higher than $80 \%$, while JPEG2000, together with REDS, presented the best results for CFs lower than $85 \%$. Indeed, one may argue that both could be employed for the entire CF range, which depends on the target application.

\section{B. Results Regarding Spectral Parameters}

Spectral parameters are important features of electromyographic records, given that meaningful information can be 


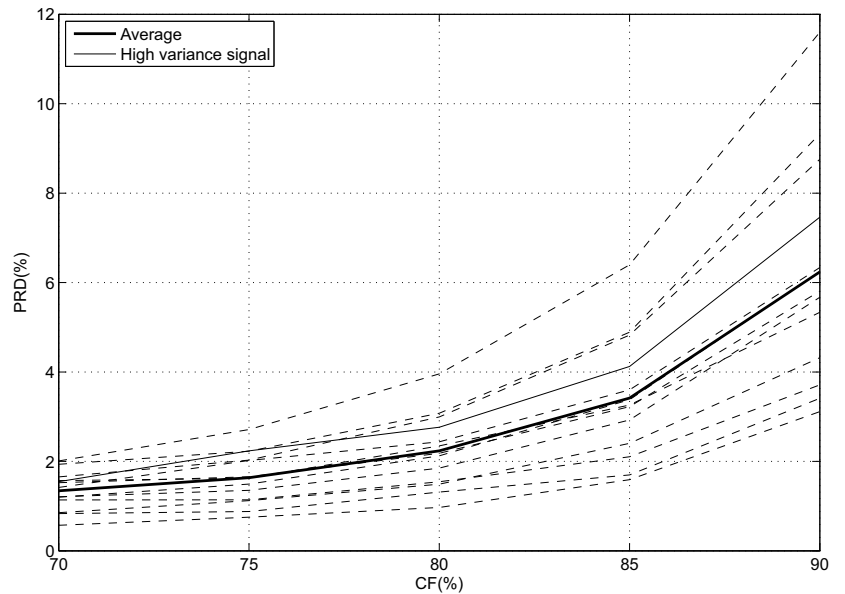

(a)

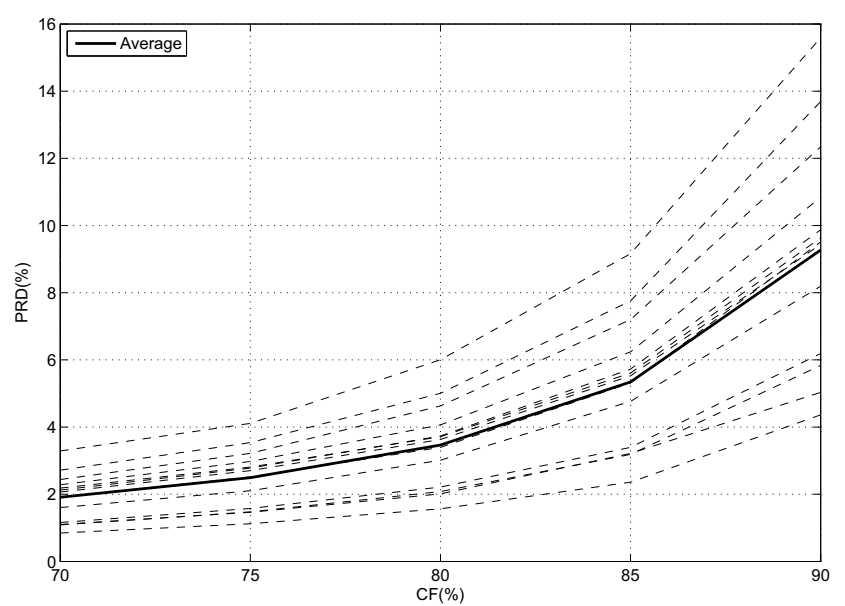

(c)

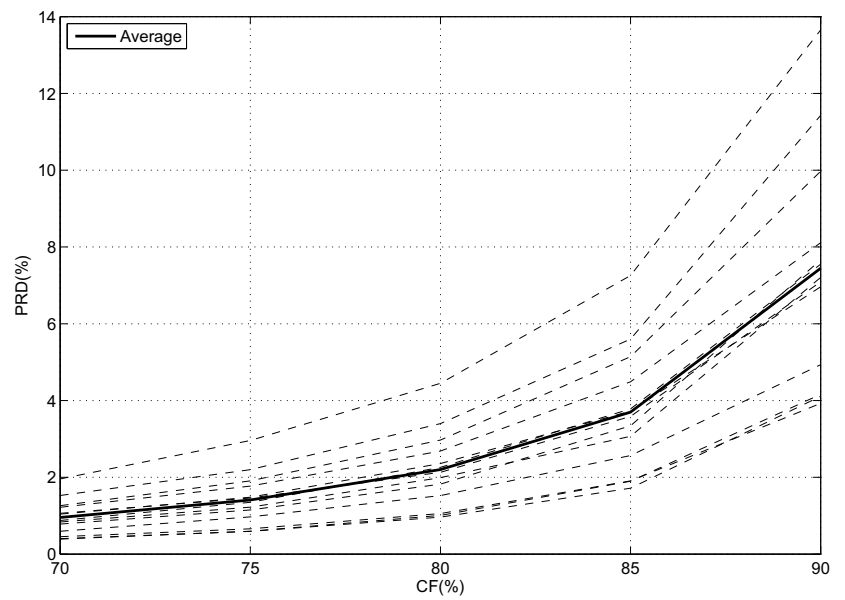

(e)

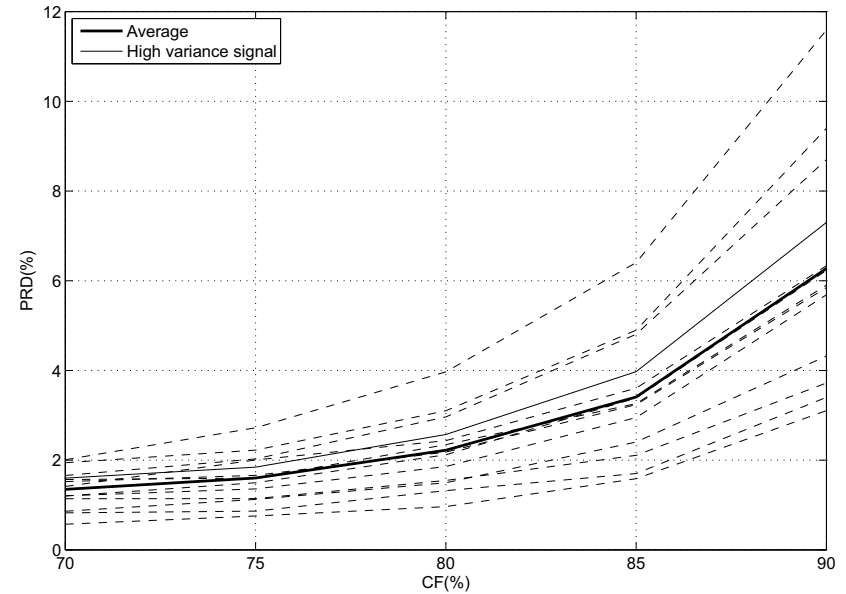

(b)

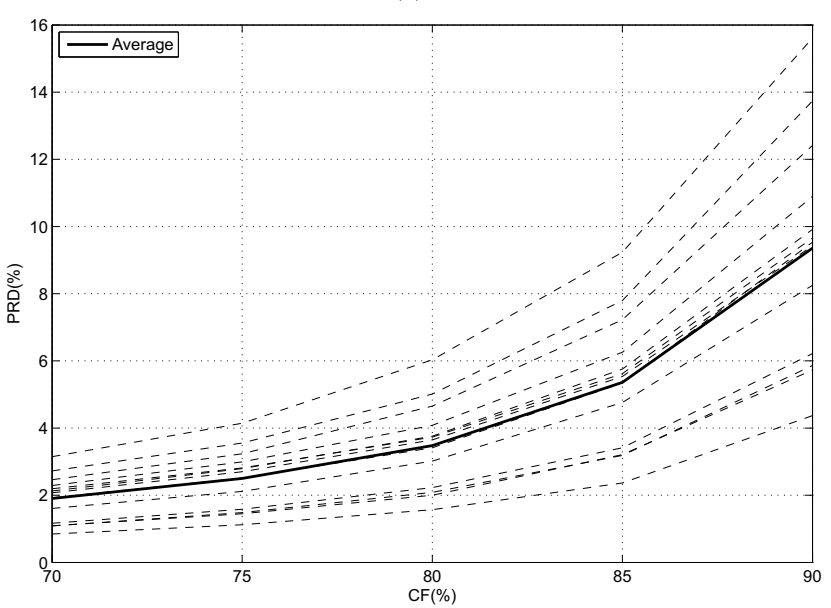

(d)

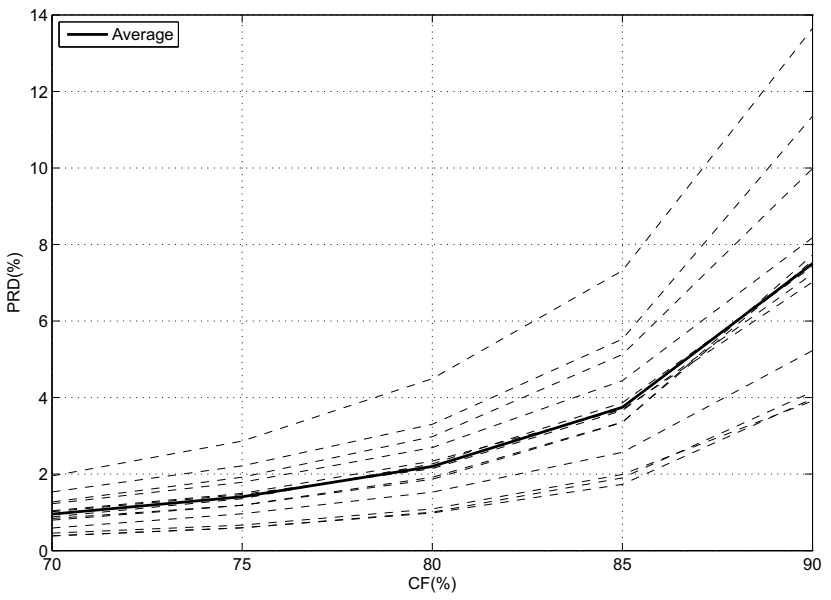

(f)

Fig. 5. Experimental results for the proposed scheme: (a) EDS + HEVC, (b) REDS + HEVC, (c) EDS + H.264/AVC, (d) REDS + H.264/AVC, (e) EDS + JPEG2000, and (f) REDS + JPEG2000.

obtained from them and used for signal analysis. For instance, the behavior regarding conduction velocity can be inferred from spectral parameters [43], during high force-level contraction, and muscle fatigue can be investigated through mean and median frequencies [44], [45]. In addition, higher-order moments can be used for estimating the waveforms of motor unit action potentials [46].
Based on what was presented above, tests were performed to verify if the proposed methodology is able to preserve the main spectral features regarding S-EMG signals, that is, mean frequency, median frequency, variance, and skewness, as done in other articles available in related the literature [31], [48]. 
TABLE III

RECONSTRUCTION ERRORS (mean percentage \pm deviation) FOR SPECTRAL PARAMETERS (CF OF $87.3 \%$ )

\begin{tabular}{cccccc}
\hline \hline Method & MVC (\%) & fmean & fmed & variance & skewness \\
\hline EDS+JPEG2000 & 60 & $0.0927 \pm 0.0886$ & $0.0255 \pm 0.0622$ & $0.3325 \pm 0.3138$ & $0.4673 \pm 0.4495$ \\
REDS+JPEG2000 & 60 & $0.1196 \pm 0.1167$ & $0.0133 \pm 0.0481$ & $0.3359 \pm 0.4617$ & $0.4429 \pm 0.4103$ \\
EDS +H.264/AVC & 60 & $0.3722 \pm 0.3052$ & $0.0240 \pm 0.0590$ & $3.8994 \pm 3.4461$ & $2.4567 \pm 2.3194$ \\
REDS+H.264/AVC & 60 & $0.3676 \pm 0.3041$ & $0.0264 \pm 0.0644$ & $3.9250 \pm 3.4577$ & $2.4873 \pm 2.3589$ \\
EDS+HEVC & 60 & $0.3436 \pm 0.3595$ & $0.0395 \pm 0.1021$ & $0.5393 \pm 0.6053$ & $0.6256 \pm 0.4902$ \\
REDS+HEVC & 60 & $0.3241 \pm 0.3504$ & $0.0395 \pm 0.1021$ & $0.5119 \pm 0.6198$ & $0.5977 \pm 0.4742$ \\
Filho et al. $[31]$ & 60 & $1.62 \pm 1.11$ & $0.98 \pm 1.24$ & $9.11 \pm 5.91$ & $56.29 \pm 21.37$ \\
Carotti et al. $[48]$ & 50 & $2.88 \pm 0.49$ & $1.83 \pm 0.88$ & - & $5.77 \pm 0.95$ \\
\hline
\end{tabular}

TABLE II

AVERAGE $P R D(\%)$ RESUltS FOR THE PROPOSED SCHEME AND ALSO

\begin{tabular}{|c|c|c|c|c|}
\hline Compression Factor & $75 \%$ & $80 \%$ & $85 \%$ & $90 \%$ \\
\hline Norris et al. [38] & 3.8 & 5 & 7.8 & 13 \\
\hline Berger et al. [27] & 2.5 & 3.3 & 6.5 & 13 \\
\hline Costa et al. (JPEG2000) [16] & 3.50 & 4.48 & 6.92 & 13.44 \\
\hline Costa et al. (H.264/AVC) [16] & 5.37 & 6.90 & 9.93 & 16.62 \\
\hline Filho et al. [31] & 1.61 & 2.51 & 4.13 & 7.36 \\
\hline Trabuco et al. [29] & 2.22 & 2.52 & 3.31 & 6.88 \\
\hline$E D S+\mathrm{HEVC}$ & 1.634 & 2.234 & 3.417 & 6.238 \\
\hline$R E D S+\mathrm{HEVC}$ & 1.601 & 2.220 & 3.408 & 6.271 \\
\hline$E D S+\mathrm{H} .264 / \mathrm{AVC}$ & 2.491 & 3.466 & 5.342 & 9.273 \\
\hline$R E D S+\mathrm{H} .264 / \mathrm{AVC}$ & 2.500 & 3.479 & 5.361 & 9.356 \\
\hline$E D S+J P E G 2000$ & 1.409 & 2.201 & 3.698 & 7.445 \\
\hline$R E D S+\mathrm{JPEG} 2000$ & 1.408 & 2.200 & 3.745 & 7.500 \\
\hline
\end{tabular}

The mean frequency is defined as

$$
f_{\text {mean }}=\frac{\sum_{i=1}^{N} f_{i} P\left[f_{i}\right]\left(f_{i}-f_{i-1}\right)}{\sum_{i=1}^{N} P\left[f_{i}\right]\left(f_{i}-f_{i-1}\right)},
$$

where $N$ is the number of frequencies used for estimating the power spectrum density (PSD) of the respective S-EMG signal. The median frequency is given by

$$
\sum_{i=1}^{f_{\text {med }}} P\left[f_{i}\right]\left(f_{i}-f_{i-1}\right)=\frac{1}{2} \sum_{i=1}^{N} P\left[f_{i}\right]\left(f_{i}-f_{i-1}\right),
$$

that is, the frequency that encompasses half signal power. Finally, variance and skewness are computed as

$$
\operatorname{var}=\sum_{i=1}^{N}\left(f_{i}-f_{\text {mean }}\right)^{2} P\left[f_{i}\right]\left(f_{i}-f_{i-1}\right),
$$

and

$$
\text { ske }=\frac{\sum_{i=1}^{N}\left(f_{i}-f_{\text {mean }}\right)^{3} P\left[f_{i}\right]\left(f_{i}-f_{i-1}\right)}{\left(\sum_{i=1}^{N}\left(f_{i}-f_{\text {mean }}\right)^{2} P\left[f_{i}\right]\left(f_{i}-f_{i-1}\right)\right)^{\frac{3}{2}}} .
$$

In order to provide spectral variables, PSD estimation through the periodogram approach [47] was applied to 1s signal epochs, considering complete S-EMG records. Table III shows the relative change in spectral variables, for a $\mathrm{CF}$ of $87.3 \%$, in form of mean percentage \pm deviation. As one may notice, the proposed scheme is able to provide percentage variation (including standard deviation) below 0.8\%, 8\%, and $5 \%$, for mean/median frequency, variance and skewness, respectively. It is worth noticing that percentage variations for all spectral variables were bellow $1 \%$, when the combination between JPEG2000 and EDS/REDS was employed.

In addition, as can be seen in Table III, the results provided by the proposed scheme outperformed the other tested algorithms. Indeed, only two articles reporting spectral parameters were found. The method presented by Filho, Silva, and Carvalho [31] operates in spatial domain, which is interesting for maintaining shape, however, such an approach causes some difficulties regarding spectral variables. Carotti et al. [48], in turn, proposed an approach based on algebraic code excited linear prediction, which captures the shape of the signal power spectrum. It is worth noticing that the results provided by Carotti et al. were obtained for an MVC of $50 \%$ (there is no result for an MVC of $60 \%$ ), however, taking into account what was presented in the same article, there should be no significant difference. Finally, the proposed approach employs transform-based encoders as back ends, which ultimately operate in frequency domain, by compacting energy in a few transform coefficients. As a consequence, even for a high CF $(87.3 \%)$, spectral parameters are accurately preserved.

\section{Results Regarding Encoding Time}

In order to provide some information regarding complexity, encoding times, for the entire database, were obtained. HM and JM software modules, which implement HEVC and H.264/AVC, respectively, already provide measurement options, whereas system functions were employed for JPEG2000. All experiments were conducted on an otherwise idle Intel Core i7-4510U, with $2.0 \mathrm{GHz}$ of clock and $8 \mathrm{~GB}$ of RAM, running Windows 8 64-bits. The obtained results, for the 13 test signals, are presented in Fig. 6.

In order to simplify the analysis, all curves, for each encoder, were also averaged and the result was plotted in Fig. 6. One may argue that the encoding time is, in average, approximately linear, regarding the target $\mathrm{CF}$ range, although some variation is noticed for JPEG2000. This is a very interesting result, since hardware restrictions, for low CFs, seem not to be an issue. In addition, JPEG2000 is more than 80 and 350 times faster than H.264 and HEVC, respectively, and the percentage increase in encoding time, regarding signal length, is less pronounced in JPEG2000.

In summary, the HEVC encoder presents the highest encoding times, while JPEG2000 is the fastest. Indeed, both HEVC and H.264/AVC employ a hybrid approach, by performing block prediction and transformation/quantization of residue signals. However, HEVC uses a more sophisticated and com- 


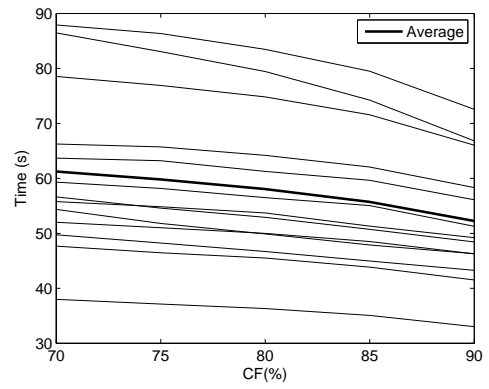

(a)

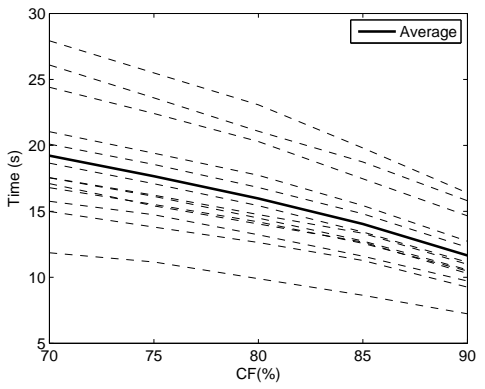

(b)

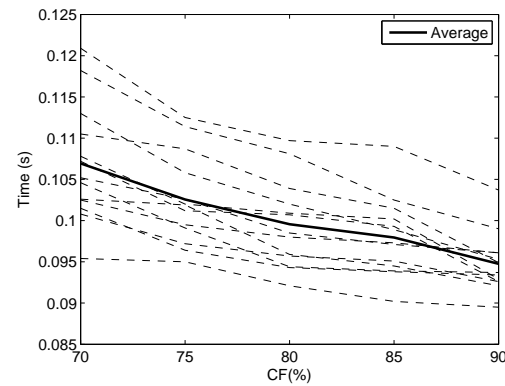

(c)

Fig. 6. Elapsed encoding time curves for (a) HEVC, (b) H.264/AVC, and (c) JPEG2000.

plex prediction procedure, when compared with H.264/AVC, which ultimately results in higher encoding times.

\section{CONCLUSION}

The proposed methodology can be considered as an effective alternative for S-EMG signal compression, with state-ofthe-art performances for compression factors of $75 \%$ and $80 \%$, when JPEG2000 is employed, and 90\%, when HEVC is chosen as back end. Indeed, the proposed scheme is effective in preserving the S-EMG signal shape, according to the resulting PRD curves. Besides, the proposed preprocessing techniques (EDS and REDS) are able to improve the exploitation of intersegment correlations, except when HEVC is used with REDS, at high compression factors, as can be seen in Table I.

During the performed experiments, which intended to validate the proposed methodology, both EDS and REDS techniques combined with JPEG2000 and HEVC gave rise to schemes that overcame state-of-the-art approaches, available in the literature. JPEG2000 is better at low CFs and HEVC at high CFs; however, given what is shown in Tables I and II, both could be used for entire CF range, depending on the target application.

In a more general approach, the use of EDS is advised, given the overall performance presented here; however, if a specific group presenting high variance must be processed, REDS may be a feasible option, at least when using HEVC, and has the potential to result in a slightly better performance.

Regarding spectral parameters, all tested combinations of preprocessing techniques and back ends were able to preserve the mean and media frequencies, with results comparable and, in many cases, even better than what was obtained by Carotti et al. [24] and Filho et al. [31]. However, regarding variance and skewness, results with H.264 and EDS/REDS are much worse than the other combinations. If the main concern is the preservation of spectral parameters, JPEG2000 and HEVC are good choices.

It is worth noticing that the proposed scheme is an interesting solution when both signal shape or spectral parameters are of paramount importance, without any algorithm tuning or adaptation. Indeed, the majority of available schemes aim to maintain signal shape [16], [26], [27], [29], [31], [40] and the ones with high performance regarding spectral parameters do not present expressive results regarding waveform errors [24], [48].
Another important decision parameter is the associated encoding time, which is related to computational complexity. The performed encoding-time tests revealed that JPEG2000 is 350 times faster than HEVC, while no test S-EMG record took more than $125 \mathrm{~ms}$ to be processed by JPEG2000, for the test configuration. If the target platform presents hardware restrictions, JPEG2000 may be a good choice, with good results for the entire $\mathrm{CF}$ range. However, when applications such as databases, which are managed by dedicated servers, or specific medical platforms are considered, HEVC may be preferred, given the possible availability coding packages and the high performance at high CFs.

It is worth noticing that the proposed scheme can be used with other image and video encoders and preprocessing techniques, that is, the present methodology may be employed together with tools suitable to specific applications.

Currently, one may argue that schemes for processing $\mathrm{S}$-EMG signals as images seem to be mature enough for commercial applications. Besides the results presented in this work, it is possible to notice that such approaches have the potential to provide fast development and dissemination, given that algorithms such as JPEG2000 and also HEVC may already be available in the target platform.

As future work, new preprocessing techniques will be developed, which can enhance both inter and intrasegment correlations, in order to further improve the performance of available image compression tools, or prioritize specific signal properties, such as spectral parameters. In addition, other biological signals will be processed through the same methodology, with preprocessing techniques suitable to their structures.

\section{ACKNOWLEDGMENT}

The authors wish to thank Professor Dr. Pedro Berger, for providing the test signals used in this work. Furthermore, part of the results presented in this paper were sponsored by Samsung Eletrônica da Amazônia Ltda., under the terms of Brazilian federal law No. 8.387/91 (SUFRAMA).

\section{REFERENCES}

[1] W. C. de Melo, E. B. L. Filho and W. S. S. Júnior, "Compressão de Sinais Eletromiográficos Baseada em Técnicas Bidimensionais," in Proc. of the XXXIII Brazilian Telecommunications Symposium, pp. 1-5, Juiz de Fora, Brazil, Sep. 2015, doi: 10.14209/sbrt.2015.66. (in Portuguese) 
[2] R. Merletti and P. Parker, Electromyography: Engineering and Noninvasive Applications. Hoboken, NJ: John Wiley \& Sons, 2004.

[3] E. Fiorucci, G. Bucci, R. Cattaneo and A. Monaco, "The Measurement of Surface Electromyographic Signal in Rest Position for the Correct Prescription of Eyeglasses," IEEE Transactions on Instrumentation and Measurement, vol. 61, no. 2, pp. 419-428, Feb. 2012, doi: 10.1109/TIM.2011.2164838.

[4] F. Farfan, J. Politti and C. Felice, "Evaluation of EMG processing techniques using Information Theory," Biomedical Engineering Online, vol. 9, no. 1, pp. 72, Nov. 2010, doi: 10.1186/1475-925X-9-72.

[5] P. Parker, K. Englehart and B. Hudgins, "Myoelectric signal processing for control of powered limb prostheses," Journal of Electromyography and Kinesiology, vol. 16, no. 6, pp. 541-548, Dec. 2006, doi: 10.1016/j.jelekin.2006.08.006.

[6] A. Smrdel and F. Jager, "Separating sets of term and pre-term uterine EMG records," Phisiological Measurement, vol. 36, no. 2, pp. 341-355, Feb. 2015, doi: 10.1088/0967-3334/36/2/341

[7] A. H. Al-Timemy, G. Bugmann, J. Escudero and N. Outram, "Classification of Finger Movements for the Dexterous Hand Prosthesis Control With Surface Electromyography," IEEE Journal of Biomedical and Health Informatics, vol. 17, no. 3, pp. 608-618, May 2013, doi: 10.1109/JBHI.2013.2249590.

[8] J. Duchłne and J.-Y. Hogrel, "A Model of EMG Generation," IEEE Transactions on Biomedical Engineering, vol. 47, no. 2, pp. 192-201, Feb. 2000, doi: 10.1109/10.821754.

[9] M. Gauthaam and S. S. Kumar, "EMG Controlled Bionic Arm," in Proc. of the National Conference on Innovations in Emerging Technology, pp. 111-114, Erode, India, Feb. 2011, doi: 10.1109/NCOIET.2011.5738813.

[10] A. J. Young and L. J. Hargrove and T. A. Kuiken, 'Improving Myoelectric Pattern Recognition Robustness to Electrode Shift by Changing Interelectrode Distance and Electrode Configuration," IEEE Transactions on Biomedical Engineering, vol. 59, no. 3, pp. 645-652, Mar. 2012, doi: 10.1109/TBME.2011.2177662.

[11] S. Sharma and M. Singh, "On Board EMG Signal Compression for Portable Devices - A Study," in Proc. of the International Conference on Machine Intelligence and Research Advancement (ICMIRA), pp. 584-588, Katra, India, Dec. 2013, doi: 10.1109/ICMIRA.2013.122.

[12] G. D. Fraser, A. D. C. Chan, J. R. Green and D.-T. MacIsaac, "Automated Biosignal Quality Analysis for Electromyography Using a OneClass Support Vector Machine," IEEE Transactions on Instrumentation and Measurement, vol. 63, no. 12, pp. 2919-2930, Apr. 2014, doi: 10.1109/TIM.2014.2317296.

[13] C. Itiki, S. S. Furuie and R. Merletti, "Compression of high-density EMG signals for trapezius and gastrocnemius muscles," BioMedical Engineering OnLine, vol. 13, pp. 25, Mar. 2014, doi: 10.1186/1475-925X13-25.

[14] E. B. L. Filho, E. A. B. da Silva, M. B. de Carvalho, W. S. S.Júnior and J. Koiller, "Electrocardiographic Signal Compression using Multiscale Recurrent Patterns," IEEE Transactions on Circuits and Systems I: Regular Papers, vol. 52, no. 12, pp. 2739-2753, Jul. 2005, doi: 10.1109/TCSI.2005.857873.

[15] T. Siiskonen and N. Päivinen, "Versatile Evaluations of Effects on DCTbased lossy compression of EMG signals on medical parameters," in Proc. of the 4th International Conference on Informatics in Control, Automation and Robotics, pp. 149-156, Angers, France, May 2007, doi: 10.5220/0001640501490156.

[16] M. V. C. Costa, J. L. A. Carvalho, P. A. Berger, A. Zaghetto and A. F. da Rocha and F. A. O. Nascimento, "Two-dimensional compression of surface electromyographic signals using column-correlation sorting and image encoders," in Proc. of the 31st Annual International Conference of the IEEE Engineering in Medicine and Biology Society, pp. 428-431, Minneapolis, USA, Sep. 2009, doi: 10.1109/IEMBS.2009.5333134.

[17] W. C. de Melo, E. B. L. Filho and W. S. S. Júnior, "Electromyographic Signal Compression Based on Preprocessing Techniques," in Proc. of the 34st Annual International Conference of the IEEE Engineering in Medicine and Biology Society, pp. 5404-5407, San Diego, USA, Aug. 2012, doi: 10.1109/EMBC.2012.6347216.

[18] D. S. Taubman and M. W. Marcellin, JPEG2000: Image Compression Fundamentals, Standards and Practice, Boston: Kluwer Academic Publishers, 2002

[19] C. Christopoulos and A. Skodras and T. Ebrahimi, "The JPEG2000 still image coding system: an overview", IEEE Transactions on Consumer Electronics, vol. 46, no. 4, pp 1103-1127, Nov. 2000, doi: $10.1109 / 30.920468$

[20] T. Wiegand, G. J. Sullivan, G. Bjontegaard and A. Luthra, "Overview of the H.264/AVC video coding standard," IEEE Transactions on Circuits and Systems for Video Technology, vol. 13, no. 7, pp. 560-576, Jul. 2003, doi: 10.1109/TCSVT.2003.815165.

[21] G. J. Sullivan, J. Ohm, W.-J. Han and T. Wiegand, "Overview of the High Efficiency Video Coding (HEVC) Standard," IEEE Transactions on Circuits and Systems for Video Technology, vol. 22, no. 12, pp. 16491668, Dec. 2012, doi: 10.1109/TCSVT.2012.2221191.

[22] J. F. Norris and D. F. Lovely, "Real-time compression of myoelectric data utilising adaptive differential pulse code modulation," Medical and Biological Engineering and Computing, vol. 33, no. 5, pp. 629-635, Dec. 1995, doi: 10.1007/BF02510779.

[23] T. Grönfors, M. reinikainen, and T. Sihvonen, "Vector quantization as a method for integer EMG signal compression," Journal of Medical Engineering \& Technology, vol. 30, no. 1, pp. 41-52, Jan. 2006, doi: 10.1080/03091900500130872.

[24] E. S. G. Carotti, J. C. de Martin, and D. Farina, "Linear predictive coding of myoelectric signals," in Proc. of the 30th IEEE International Conference on Acoustics, Speech, and Signal Processing, vol. 5, pp. 629632, Philadelphia, USA, Mar. 2005, doi: 10.1109/ICASSP.2005.1416382.

[25] A. P. Guerrero and C. Mailhes, "On the choice of an electromyogram data compression method," in Proc. of the 19th Annual International Conference of the IEEE Engineering in Medicine and Biology Society, vol. 4, pp. 1558-1561, Chicago, USA, Oct. 1997, doi: 10.1109/IEMBS.1997.757009.

[26] J. A. Norris, K. Englehart, and D. Lovely, "Myoelectric signal compression using zero-trees of wavelet coefficients," Medical Engineering \& Physics, vol. 25, no. 9, pp. 739-746, Nov. 2003, doi: 10.1016/S13504533(03)00118-8

[27] P. A. Berger, F. A. O. Nascimento, J. C. Carmo, and A. F. Rocha, "Compression of EMG signals with wavelet transform and artificial neural networks," Institute of Physics Publishing: Physiological Measurement, vol. 27, no. 6, pp. 457-465, Jun. 2006, doi: 10.1088/0967-3334/27/6/003.

[28] N. Jain and R. Vig, "Wavelet based vector quantization with tree code vectors for EMG Signal compression," in Proc. of the 6th WSEAS International Conference on Signal Pprocessing, pp. 1-8, Dallas, USA, Mar. 2007.

[29] M. H. Trabuco, M. V. C. Costa and F. A. O. Nascimento, "S-EMG signal compression based on domain transformation and spectral shape dynamic bit allocation," BioMedical Engineering OnLine, vol. 13, pp. 22, Feb. 2014, doi: 10.1186/1475-925X-13-22.

[30] M. H. Trabuco, M. V. C. Costa, F. P. Schwartz and F. A. O. Nascimento, "Compression of S-EMG signals by transforms and spectral profile for bit allocation," in Proc. of the Pan American Health Care Exchanges (PAHCE), pp. 1-6, Medellin, Colombia, Apr. 2013, doi: 10.1109/PAHCE.2013.6568316.

[31] E. B. L. Filho, E. A. B. Silva and M. B. Carvalho, "On EMG Signal Compression with Recurrent Patterns," IEEE Transactions on Biomedical Engineering, vol. 55, no. 7, pp. 1920-1923, Jul. 2008, doi: 10.1109/TBME.2008.919729.

[32] C. Yi and Y. Tian, "Text String Detection From Natural Scenes by Structure-Based Partition and Grouping," IEEE Transactions on Image Processing, vol. 20, no. 9, pp. 2594-2605, Mar. 2011, doi: 10.1109/TIP.2011.2126586.

[33] K. Nazarpour, A. R. Sharafat and S. M. Firoozabadi, "Negentropy Analysis of Surface Electromyogram Signal," in Proc. of the 13th IEEE Workshop on Statistical Signal Processing, pp. 974-977, Novosibirsk, Russia, Jul. 2005, doi: 10.1109/SSP.2005.1628736.

[34] J. Lainema, F. Bossen, W.-J. Han, J. Min and K. Ugur, "Intra Coding of the HEVC Standard," IEEE Transactions on Circuits and Systems for Video Technology, vol. 22, no. 12, pp. 1792-1801, Dec. 2012, doi: 10.1109/TCSVT.2012.2221525.

[35] A. Moffat, R. Neal and I. H. Witten, "Arithmetic coding revisited," ACM Transactions on Information Systems, vol. 16, pp. 256-294, Jul. 1998, doi: 10.1145/290159.290162.

[36] M. T. Pourazad, C. Doutre, M. Azimiand and P. Nasiopoulos, "HEVC: The New Gold Standard for Video Compression: How Does HEVC Compare with H.264/AVC?," IEEE Consumer Electronics Magazine, vol. 1, no. 3, pp. 36-46, Jul. 2012, doi: 10.1109/MCE.2012.2192754.

[37] T. Nguyen and D. Marpe, "Objective Performance Evaluation of the HEVC Main Still Picture Profile," IEEE Transactions on Circuits and Systems for Video Technology, vol. 25, no. 5, pp. 790-797, May 2015, doi: 10.1109/TCSVT.2014.2358000.

[38] J. A. Norris, K. Englehart, and D. Lovely, "Steady-state and dynamic myoelectric signal compression using embedded zero-tree wavelets," in Proc. of the 23rd Annual International Conference of the IEEE Engineering in Medicine and Biology Society, pp.1879-1882, Buenos Aires, Argentina, Oct. 2001, doi: 10.1109/IEMBS.2001.1020592. 
[39] E. S. G. Carotti, J. C. De Martin, R. Merletti and D. Farina, "Compression of surface EMG signals with algebraic code excited linear prediction," in Proc. of the IEEE International Conference on Acoustic, Speech and Signal Processing, vol. 3, pp. 1148-1151, Toulouse, France, May 2006, doi: 10.1109/ICASSP.2006.1660862.

[40] M. V. C. Costa, P. A. Berger, A. F. da Rocha, J. L. A. Carvalho, and F. A. O. Nascimento, "Compression of Electromyographic Signals Using Image Compression Techniques," in Proc. of the 30th Annual International Conference of the IEEE Engineering in Medicine and Biology Society, pp. 2948-2951, Vancouver, Canada, Aug. 2008, doi: 10.1109/IEMBS.2008.4649821.

[41] V. Sze, M. Budagavi, and G. J. Sullivan, High Efficiency Video Coding (HEVC): Algorithms and Architectures. Springer, 2014, doi: 10.1007/9783-319-06895-4.

[42] S. Banerjee and B. L. Evans, "Tuning JPEG2000 image compression for graphics regions," in Proc. of the 5th IEEE Southwest Symposium on Image Analysis and Interpretation, pp. 67-71, Santa Fe, USA, Apr. 2002, doi: 10.1109/IAI.2002.999891.

[43] H. Broman, G. Bilotto, and C. J. de Luca, "Myoelectric signal conduction velocity and spectral parameters: influence of force and time," Journal of Applied Physiology, vol. 58, no. 5, pp. 1428-1437, May 1985.

[44] B. Elfvinga, G. Németha, I. Arvidssonb, and M. Lamontagne, "Reliability of EMG spectral parameters in repeated measurements of back muscle fatigue," Journal of Electromyography and Kinesiology, vol. 9, no. 4, pp. 235-243, Aug. 1999, doi: 10.1016/S1050-6411(98)00049-2.

[45] S. Thongpanja, A. Phinyomark, P. Phukpattaranont and C. Limsakul, "Mean and Median Frequency of EMG Signal to Determine Muscle Force based on Time-Dependent Power Spectrum," Elektronika ir Elektrotechnika, vol. 19, no. 3, pp. 51-56, Feb. 2013, doi: 10.5755/j01.eee.19.3.3697.

[46] M. B. I. Reaz, M. S. Hussain, and F. Mohd-Yasin, "Techniques of EMG signal analysis: detection, processing, classification and applications," Biological Procedures Online, vol. 8, no. 1, pp. 11-35, Dec. 2006, doi: $10.1251 / \mathrm{bpo} 115$.

[47] P. S. R. Diniz, E. A. B. da Silva, and S. L. Netto, Digital Signal Processing: System Analysis and Design, 2010: Cambridge University Press.

[48] E. Carotti, J. C. De Martin, R. Merletti, and D. Farina, "Compression of surface EMG signals with algebraic code excited linear prediction," Medical Engineering \& Physics, Vol. 29, No. 2, pp. 253-258, May 2006, doi: 10.1016/j.medengphy.2006.03.004.

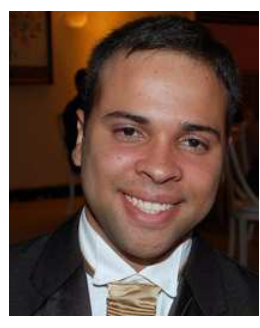

Wheidima Carneiro de Melo was born in Manaus, AM, Brazil, in 1983. He received his B. Sc. degree in electrical engineering, in 2007, from Federal University of Amazonas (UFAM), and the M. Sc. degrees in Electrical Engineering, in 2014, from Federal University of Amazonas (UFAM). Since 2013, he has been with the Department of Electrical Engineering, Superior School of Technology (EST) of Amazonas State University (UEA), as professor. His research interests include image/video processing, channel/source coding, and digital signal processing.

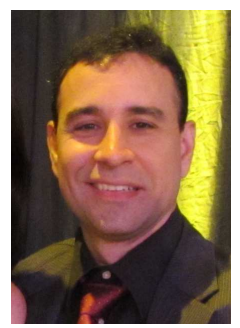

Eddie Batista de Lima Filho was born in Manaus, AM, Brazil, in 1977. He received the B. Sc. degree in Electrical Engineering from Federal University of Amazonas (UFAM), Manaus, AM, Brazil, in 1999, and the M. Sc. and D. Sc. degrees in Electrical Engineering from Federal University of Rio de Janeiro (COPPE/UFRJ), Rio de Janeiro, RJ, Brazil, in 2004 and 2008, respectively. During 2006, he worked at the Interuniversity Microelectronics Center (IMEC), Leuven, Belgium, with digital television and WiMAX MAC and PHY layers simulators. Since 2016, he has been with FPF Tech, Manaus, AM, Brazil, working with digital TV and embedded systems. His research interests include digital signal processing, channel/source coding, video/image processing, and cognitive radio.

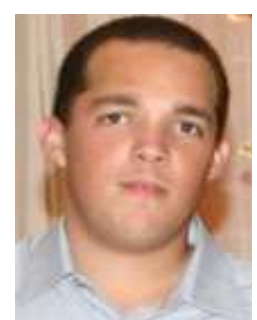

Waldir S. S. Júnior received the B.S. degree in Electrical Engineering at the Federal University of Amazonas (UFAM), Manaus, AM, Brazil, in 2000, and the M.Sc. degree in Electrical Engineering at the Federal University of Rio de Janeiro (COPPE/UFRJ), Rio de Janeiro, RJ, Brazil, in 2004 and D.Sc. degree in Electrical Engineering at the Federal University of Rio de Janeiro (COPPE/UFRJ), Rio de Janeiro, RJ, Brazil, in 2010. Since 2006, he has been with the Federal University of Amazonas (UFAM), as professor. His research interests are in the fields of pattern recognition, data compression, and digital signal processing. 